\title{
Who will clone a chromosome?
}

\author{
The cloning of factor VIII, on which most haemophiliacs depend, is a technical triumph without parallel, \\ but has left important questions unanswered. They rest for others.
}

By sad coincidence, this week there may be special interest in the publication of four long articles (pp.326 et seq.) describing how two groups in the United States have been able to isolate the gene responsible for human factor VIII, one of the essential components of the blood-clotting system. The significance of this development is described on p.326 by Professor George Brownlee and Dr Charles Rizza.

They explain that Genentech and the Genetics Institute have been able to clone the gene for factor VIII, to work out its nucleotide sequence, to infer the way in which the corresponding very large protein molecule is put together from amino acids and even to devise a system by means of which factor VIII may eventually be manufactured in large quantities.

This is also the week in which people in Australia have been startled by the news of the death of at least three infants as a consequence of tranfusion with blood now suspected of being contaminated with the virus (called HTLVIII) responsible for acquired immune deficiency syndrome (AIDS), and in which a British haemophilia patient died from what appear to have been similar causes, the contamination of supposedly pure factor VIII by the same virus.

Although people in the United States have been familiar with such tragic happenings for at least two years, the spread of this bad news is a chilling reminder both of the dangers to us all embodied by the virus responsible for AIDS and of the urgency of the need for a process for producing factor VIII that may be free from risks of that kind. It is especially cruel that people suffering from haemophilia, already a sufficient hazard to peace of mind, should now be exposed to a further risk over which they have literally no control. Whatever the commercial interests of those responsible for this week's reports, their fleetness of foot will earn full-throated congratulation. Such is the promise of what we call genetic engineering.

Unfortunately, this week's clutch of papers will also help to give that field a less enviable reputation. In the application of molecular biology to practical affairs, it is for the time being clear that bigger battalions move faster. So is there a danger, already explicit in high-energy physics, that the list of the names of the authors of a scientific article will be longer than a reasonably succinct listing of its conclusions? Readers must judge for themselves, recognizing as they do so the extraordinary character of what has been accomplished.

Hitherto, even the natural site at which factor VIII is synthesized has not been known (but the liver was always a candidate), the protein molecule is the largest whose genetic origin has yet been characterized and the circumstance that the protein (but not, yet, the glycosylation parts that naturally go with it) can actually be manufactured on a laboratory scale would not have been thought possible even at the beginning of this year.

Two sceptics' questions nevertheless arise, the first of which is whether, in the single-minded pursuit of something of undoubted value, those responsible for this staggering exploit may have neglected tantalizing scientific issues that could in the end turn out to be more interesting. And, perhaps, even more important.

Here is an example. To many readers, the most arresting feature of the stack of papers now published will be the information, mentioned almost in passing by both groups, that part of the factor VIII molecule now synthesized resembles in its amino acid sequence part of the molecule of a quite unrelated protein, the plasma protein called ceruloplasmin, whose function is for the time being largely unknown. Ceruloplasmin has two unambiguous properties - it is blue in colour (which is what its name implies) and it binds copper atoms (between four and six per molecule). But properly supplied with copper, ceruloplasmin is an oxidizing enzyme. The common guess is that its function in the human plasma is to convert ferrous $\left(\mathrm{Fe}^{2+}\right)$ into ferric $\left(\mathrm{Fe}^{3+}\right)$ ions. But nobody seems to have conclusive proof.

So why, teleologically speaking, should factor VIII resemble such a molecule? There are obviously several candidate answers. Perhaps factor VIII, like ceruloplasmin, also binds copper atoms in ways that are important to its function. (Molecules of factor $\mathrm{V}$, the analogous component of the blood-clotting system, have at least one copper-binding site.) As yet, it seems, nobody has checked.

It is also, however, possible that evolution, always ingenious, may have hit on a way of using a pre-existing molecular structure (ceruloplasmin) as a way of helping blood to clot rather than its normal function, for the sake of argument that defying the second law of thermo- dynamics, by banishing ferrous ions when they would normally occur. (Ferrous ions are an obvious threat to living things; ferrous chloride is virtually insoluble, they form free radicals easily.) The trouble is that is nobody, even now (and after reading pp.326 et seq.) can know whether factor VIII is a copper binding protein. Factor V is, but that could be a different matter.

In normal times, if the field were stagnant, these speculations would have been suitable material for a successful grant application (or several). So too would have been some of the other questions not merely unanswered but unasked by pp.326 et seq. Why (or, rather, how) does it come about that such a basic system as that responsible for the clotting of blood should require not one enzyme but several? (Because evolution has found it to be worthwhile, at the margin as the economists would say, to win a little extra efficiency at the cost of a great deal of extra elaboration, perhaps because the molecules required for these marginal purposes were already in essence available.) Why are the components of the blood-clotting system distributed so widely on the human chromosomes? (The same answer applies; they are where they happened to be, so to speak.)

But how does factor VIII function? What is the mechanism by which, in human plasma (and in association with another shadowy plasma protein, von Willebrand's factor) it catalyses the tranformation of factor Xa? Nobody has begun to guess what may be at the bottom of this process. Nobody can be blamed, for even a few months ago the question would have seemed insufferably impertinent. Yet it needs to be answered, and quickly.

That raises the second question about the balance of this triumphant effort in the cloning of factor VIII that may yet come round to haunt the cloners - is it feasible to think of manufacturing by the kilo a molecule as large as that of factor VIII without risking that it will emerge corrupted, or as bad, ineffective, from the production line? Obviously, the genetic engineers would prefer to be manufacturing a simpler molecule, one related to the molecule whose structure had now been described, as a few amino acids in an antigenic molecule may represent the biological activity of the entire structure. Will that be feasible? Time, at the worst, will tell. Otherwise, a little research may make the question answerable.

John Maddox 